BULL. AUSTRAL. MATH. SOC.

VOL. $4(197 \mid), 343-348$.

\title{
Just non-finitely-based varieties of groups
}

\author{
M. F. Newman
}

\begin{abstract}
A variety of groups is just non-finitely-based if it does not have a finite basis for its laws while all its proper subvarieties do have a finite basis. Recent work of Ol'šanskiY, Vaughan-Lee and Adjan guarantees the existence of at least one just non-finitely-based variety. In this note an infinite number of just non-finitely-based varieties are shown to exist by proving that for every prime $p$ there is a non-finitely based variety of p-groups.
\end{abstract}

A variety of groups which does not have a finite basis for its laws contains, by a routine application of Zorn's Lemma, a variety which is minimal with respect to not having a finite basis for its laws. Call such a minimal variety just non-finitely-based. The previous sentence can then be rewritten: every non-finitely-based variety contains a just non-finitely-based variety.

It follows from Vaughan-Lee's work in [5] that the product variety $\underline{\underline{T}}_{2} \underline{\underline{T}}_{2}$ (where $\underline{\underline{T}}_{2}$ is the variety generated by the dihedral group of order 8), even the subvariety defined by the additional word $\left[\left[x_{1}, x_{2}, x_{3}\right],\left[x_{4}, x_{5}, x_{6}\right],\left[x_{7}, x_{8}\right]\right]$, contains a just non-finitely-based variety. Conceivably there is only one just non-finitely-based variety in $\underline{\underline{\mathrm{T}}} \underline{\underline{\mathrm{T}}}_{2}$. Although the non-finitely-based varieties described by 01 'sanski

Received 23 November 1970. Done while holding a post-doctoral fellowship in the University of Illinois at Champaign-Urbana. The author is indebted to Dr M.R. Vaughan-Lee for sending him a preprint of his paper [5] which inspired and served as a model for this work. 
[4] and Adjan [1] are quite different from Vaughan-Lee's, they don't seem to guarantee the existence of even one more just non-finitely-based variety.

The purpose of this note is to prove:

There are an infinite number of just non-finitely-based varieties of groups.

This is done by proving for each prime $p$ that there is a variety of p-groups which does not have a finite basis for its laws. Unexplained notation is used as described in Hanna Neumann's book [3].

In the word group $W$ on the set $\left\{x, y, z, x_{1}, x_{2}, \ldots\right\}$ let $u(k)=\left[x_{1}, x_{2}\right] \ldots\left[x_{2 k-1}, x_{2 k}\right]$ and $v(k)=\left[[x, y, z],[x, y, z]^{u(k)}, \ldots,[x, y ; z]^{u(k)^{p-1}}\right]$.

It suffices to prove that there is a group in the variety $\left(\underline{A}_{p} \underline{A} p \wedge \stackrel{\mathrm{N}}{=}\right)_{\underline{\mathrm{T}}}$ in which $v(1), \ldots, v(n-1)$ are laws but $v(n)$ is not a law (here for $p$ odd $\stackrel{\mathrm{T}}{\mathrm{p}}_{p}$ is the variety generated by the non-abelian group of order $p^{3}$ and exponent $p$ ); for then a standard argument shows that the subvariety of $(\stackrel{A}{A} \stackrel{A}{\sim} \stackrel{N}{=}) \underset{p}{\underline{T}}$ consisting of the groups in which $v(1), v(2), \ldots$ are all laws cannot have a finite basis for its laws.

Let $A$ be a free group in $\underset{p}{\mathrm{~T}}$ of finite rank $m$. Let $B$ be a free group of $\stackrel{A}{A} \Rightarrow p^{A} \stackrel{\mathbb{N}}{p}_{p}$ of rank the order of $A$ freely generated by $\left\{b_{a}: a \in A\right\}$. There is a natural action of $A$ on $B$ described by $\left(b_{a}\right)^{a^{\prime}}=b_{a \alpha^{\prime}}$. Let $C$ be the splitting extension of $B$ by $A$ with this action. As usual $A$ and $B$ will be identified with the appropriate subgroups of $C$, and the elements of $C$ will be taken to have the form $a b$ with $a$ in $A$ and $b$ in $B$ (identity elements will be omitted). The required group will be exhibited as a factor group of $C$.

The order of the commutator subgroup $A^{\prime}$ of $A$ is $p^{m(m-1) / 2}$. The number of elements of $A^{\prime}$ which can be written as the product of $n-1$ commutators is at most $p^{2 m(n-1)}$ (since the identity $e$ is a commutator 
this covers products of less than $n-1$ commutators as well). Take $m \geq 4 n$, then there is an element, $d$ say, of $A^{\prime}$ which can be written as the product of $n$ commutators but not of $n-1$ (or fewer) commutators. Let $D$ denote the subgroup generated by $d$.

The $p$-th term $\gamma_{p}(B)$ of the lower central series of $B$ is an elementary abelian p-group generated (not freely) by the elements $\left[b_{a_{1}}, \ldots, b_{a_{p}}\right]$ which will be written $\left[\left[a_{1}, \ldots, a_{p}\right]\right]$ from now on. The result will be proved by exhibiting a subgroup $R$ of $\gamma_{p}(B)$ which contains all the values in $c$ of the words $v(1), \ldots, v(n-1)$ but not every value of $v(n)$; this is enough for then the verbal subgroup $V$ of $C$ corresponding to the set $\{v(1), \ldots, v(n-1)\}$ of words $l i$ es in $R$ and consequently $C / V$ will have the required properties.

Let $M$ be the subgroup of $\gamma_{p}(B)$ generated by the $\left[\left[a_{1}, \ldots, a_{p}\right]\right]$ where $\left\{a_{1}, \ldots, a_{p}\right\}$ is a coset of $D$ and $L$ the subgroup generated by all the other $\left[\left[a_{1}, \ldots, a_{p}\right]\right]$. Every relation between the $\left[\left[a_{1}, \ldots, a_{p}\right]\right]$ is a consequence of relations of the types (*) $^{*}\left\{\begin{array}{l}{\left[\left[a_{1}, a_{2}, a_{3}, \ldots\right]\right]\left[\left[a_{2}, a_{3}, a_{1}, \ldots\right]\right]\left[\left[a_{3}, a_{1}, a_{2}, \ldots\right]\right]=e,} \\ {\left[\left[a_{1}, a_{2}, \ldots, a_{i}, a_{i+1}, \ldots\right]\right]\left[\left[a_{1}, a_{2}, \ldots, a_{i+1}, a_{i}, \ldots\right]\right]^{-1}=e,} \\ \text { and }\left[\left[a_{1}, a_{2}, \ldots\right]\right]\left[\left[a_{2}, a_{1}, \ldots\right]\right]=e,\end{array}\right.$ so $\gamma_{p}(B)$ is the direct product of $M$ and $L$. Let $U$ be a transversal of $D$ in $A^{\prime}$ which contains $e$, and $T$ a transversal of $A^{\prime}$ in $A$ which contains $e$. The subgroup $M$ is freely generated by the $g(t, u, i)=\left[\left[t_{u} d^{i}, t u, t u d, \ldots, t u d^{i-1}, t u d^{i+1}, \ldots, t u d^{p-1}\right]\right]$ where $t, u$ run through $T, U$ respectively and $i$ through $\{1, \ldots, p-1\}$ because these commutators are basic in any order in which $t u<t u d<\ldots<t u a^{p-1}$ for all $t, u$ (see for instance 4.05 of [2] for a direct proof). Let $g_{t}=g(t, e, 1)$. Let $P$ be the subgroup of $M$ 
generated by the $g_{t}$ with $t$ running through $T$, and let $N$ be the subgroup of $M$ generated by all the products $g(t, u, i) g_{t}^{-i}$. Clearly $M$ is the direct product of $P$ and $N$. Take $R$ to be the direct product of $N$ and $L$.

Let $\theta$ be a homomorphism from the word group $W$ to $C$ such that $x \theta=b_{e}, y \theta=t, z \theta=t^{\prime}$, where $t, t^{\prime}$ are distinct elements of $T \backslash\{e\}$, and $u(n) \theta=d$, then $v(n) \theta=g_{e}^{-1} g_{t} g_{t} g_{t t^{\prime}}^{-1}$ with $r$ in $R$. Thus $v(n) \theta$ does not belong to $R$, and one of the claims is established.

The following observation will be needed in proving the other claim. For all $a_{1}, \ldots, a_{p}$ in $A$ and $h$ in $A^{\prime}$, $\left[\left[a_{1}, \ldots, a_{p}\right]\right]^{h}=\left[\left[a_{1} \hbar, \ldots, a_{p} h\right]\right]=\left[\left[a_{1}, \ldots, a_{p}\right]\right]$ for some $r$ in $R$ because if $\left\{a_{1}, \ldots, a_{p}\right\}$ is not a coset of $D$ neither is $\left\{a_{1} h, \ldots, a_{p} h\right\}$, while if $\left\{a_{1}, \ldots, a_{p}\right\}$ is the coset tuD and $a_{1}=t u d^{i}, a_{2}=t u d^{j}$, then both $\left[\left[a_{1}, \ldots, a_{p}\right]\right]$ and $\left[\left[a_{1} h, \ldots, a_{p} h\right]\right]$ are congruent to $g_{t}^{i-j}$ modulo $N$. Hence, by linearity, $\left[b_{1}, \ldots, b_{p}\right]^{h}$ is congruent to $\left[b_{1}, \ldots, b_{p}\right]$ modulo $R$ for all $b_{1}, \ldots, b_{p}$ in $B$ and $h$ in $A^{\prime}$.

Every value of $v(k)$ for $k$ in $\{1, \ldots, n-1\}$ has the form $\left[b, b^{c}, \ldots, b^{c^{p-1}}\right]$ where $b$ belongs to $B$ and $c$ is a product of at most $n-1$ commutators from $A^{\prime}$. All these elements will be shown to belong to $R$. It suffices to prove for $b_{1}, b_{2}$ in $B$ and $h$ in $A^{\prime}$ that $\left[b_{1} b_{2},\left(b_{1} b_{2}\right)^{h}, \ldots,\left(b_{1} b_{2}\right)^{h^{p-1}}\right]$ is congruent to $\left[b_{1}, b_{1}^{h}, \ldots, b_{1}^{h^{p-1}}\right]\left[b_{2}, b_{2}^{h}, \ldots, b_{2}^{h^{p-1}}\right]$ modulo $R$, for then $\left[b, b^{c}, \ldots, b^{c^{p-1}}\right]$ is, by induction on the length of $b$ as a product of generators $b_{a}$, congruent modulo $R$ to a product of commutators 
$\left[b_{a}, b_{a}^{c}, \ldots, b_{a}^{c^{p-1}}\right]$ each of which lies in $L$ because $\left\{a, a c, \ldots, a c^{p-1}\right\}$ is not a coset of $D$. Now $\left[b_{1} b_{2},\left(b_{1} b_{2}\right)^{h}, \ldots,\left(b_{1} b_{2}\right)^{h^{p-1}}\right]=\prod\left[b_{f(0)}, b_{f(1)}^{h}, \ldots, b_{f(p-1)}^{h^{p-1}}\right]$ where $f$ runs through the set $F$ of functions from $\{0, \ldots, p-1\}$ to $\{1,2\}$. Define a mapping $\tau$ on $F$ by

$$
f \tau(i)=f(i+1) \text { for all } i \text { (taking }(p-1)+1=0) \text {. }
$$

The orbits of $\tau$ in $F$ are all of length $p$ except for two of length $I$ corresponding to the two constant functions. The desired result comes by proving $b^{*}=\prod_{j=0}^{p-1}\left[b_{f \tau^{j}(0)}, b_{f \tau^{j}(1)}^{h}, \ldots, b^{h h^{p-1}}\right]$ belongs to $R$ for all $f$ in $F$. Now by the definition of $\tau$, $b^{*}=\prod_{j=0}^{p-1}\left[b_{f(j)}, b_{f(j+1)}^{h}, \ldots, b_{f(j-1)}^{h^{p-1}}\right]$. It follows from the observation in the last paragraph that $b^{*}=\prod_{j=0}^{p-1}\left[b_{f(j)}^{h^{j}}, \ldots, b_{f(j-1)}^{h^{j-1}}\right] r$ for some $r$ in $R$. Use of relations of the type (*) yields $b^{*}=r$ and the proof is complete.

\section{References}

[1] S.1. Adjan, "Infinite irreducible systems of group identities" (Russian), Dokl. Akad. Nauk SSSR 190 (1970), 499-501; Soviet Math. Dokz. 11 (1970), 113-115.

[2] L.G. Kovács and M.F. Newman, "On non-Cross varieties of groups", $J$. Austral. Math. Soc. 12 (1971), 129-144.

[3] Hanna Neumann, Varieties of groups (Ergebnisse der Mathematik und ihrer Grenzgebiete, Band 37, Springer-Verlag, Berlin, Heidelberg, New York, 1967).

[4] A.Ju. Ol'šanskiY, "On the problem of a finite basis for the identities of groups" (Russian), Izv, Akad. Nauk SSSR 34 (1970), 376-384. 
[5] M.R. Vaughan-Lee, "Uncountably many varieties of groups", BuZZ. London Math. Soc. 2 (1970), 280-286.

Institute of Advanced Studies, Australian National University, Canberra, ACT. 